\title{
Abandono en carreras de Ingeniería. Un estudio de los aspectos académicos, socio- demográficos, laborales y vitales ${ }^{1}$
}

\author{
Drop-outs in Engineering courses \\ A study of the academic, socio-demographic, vital and work aspects
}

\author{
Abandono em cursos de engenharia. Uma análise de aspectos \\ acadêmicos, sociodemográficos, trabalhistas e vitais
}

ISSN 1688-9304 - DOI: https://doi.org/10.18861/cied.2019.10.2.2908

Jacqueline Elizabet Moreno*2

https://orcid.org/0000-0002-1176-7149

Analía Claudia Chiecher*3

https://orcid.org/0000-0002-5421-6865

Fecha de recibido: 18/12/2018

Fecha de aprobado: 7/05/2019

\section{Resumen}

El estudio que presenta este artículo propone reconstruir retrospectivamente las trayectorias académicas de una cohorte de estudiantes de ingeniería contactada cinco años luego de su ingreso a la universidad. En el momento del relevamiento se constató que, en el lapso de los 5 años transcurridos desde su ingreso, la mitad de la cohorte había abandonado los estudios. Interesa en este escrito estudiar el grupo de sujetos que abandonaron los estudios y analizar, en sus trayectorias personales, factores vinculados al contexto familiar, la trayectoria laboral e historia de vida de los sujetos, además de los motivos del abandono. Este grupo de estudiantes que abandonaron sus estudios a los 5 años está conformado por 80 sujetos. Se dispone de datos sociodemográficos del grupo total, en tanto que fue posible tomar contacto con 18 sujetos a quienes se administró una encuesta longitudinal de reconstrucción de trayectorias así como una entrevista semiestructurada. Los resultados más destacados muestran que, en relación con los aspectos sociodemográficos, la mayoría de los sujetos llegaron desde otras localidades para iniciar sus estudios universitarios y sus padres han tenido acceso solo a niveles educativos bajos. En cuanto a la situación laboral, el porcentaje de estudiantes que informaron trabajar y estudiar en paralelo no es mayoritario. Sin embargo, se advierte que en no pocas ocasiones el momento de inicio de las actividades laborales coincide con el del inicio de los estudios universitarios. Por último, los acontecimientos vitales que los estudiantes han percibido como influyentes en sus trayectorias educativas son variados. No obstante, profundizando en los motivos que llevaron a estos jóvenes a abandonar sus estudios encontramos que la mayoría atribuye el abandono a aspectos vinculados con la elección vocacional y con dificultades en el orden de lo académico.

Palabras clave: trayectorias académicas - abandono - características sociodemográficas - contexto familiar - trayectoria laboral - acontecimientos vitales.

\footnotetext{
Abstract

The study presented in this article proposes a retrospective reconstruction of academic performance in a cohort of engineering students who were contacted five years after their admission to university. At the time of the survey -5 years from the date of their admission-,
} 
it was found that half of the cohort had abandoned their studies. This article discusses on the group of subjects who had abandoned studies and analyses those aspects related to their family context, their work experience, and life history of each of them, as well as the reasons for dropping out.

A total of 80 subjects constitute the group who started an engineering course and dropped out within 5 years. Socio-demographic data are available for the entire group, whereas for 18 subjects, it was also possible to administer a semi-structured interview as well as a longitudinal survey to reconstruct their academic performance. The most outstanding results show that, in relation to socio-demographic aspects, most of the subjects came from other localities to start their university studies and their parents had had only access to low educational levels. Regarding employment, the percentage of students who reported working and studying in parallel is not the majority. However, it is noted that on several occasions the start date of employment coincides with the time they began studying at university. Finally, the life events that students have perceived as being influential in their educational trajectories are varied. Nevertheless, by delving deeper into the reasons that led these young people to abandon their studies, we find that most of them account their abandonment to aspects linked to vocational choice and to academic difficulties.

Keywords: academic trajectories - drop-out rates - socio-demographic aspects - family context - work history - personal hallmarks.

\section{Resumo}

A pesquisa apresentada nesse artigo propõe reconstruir retrospectivamente as trajetórias acadêmicas de uma coorte de estudantes de engenharia contatados cinco anos após ingressar na universidade. Em ocasião da pesquisa, verificou-se que metade da coorte havia abandonado a escola no decorrer de cinco anos após a admissão. Interessa nesse artigo focalizar um grupo de sujeitos que abandonaram os estudos e analisar as implicações que em suas trajetórias tiveram aspectos relacionados ao contexto familiar, trajetória de trabalho e história de vida dos sujeitos, além dos motivos do abandono.

Um total de 80 indivíduos compõem o grupo daqueles que iniciaram um curso de engenharia e o abandonaram no decorrer de 5 anos. Os dados sociodemográficos do grupo total estão disponíveis, inclusos 18 indivíduos desse grupo com os quais foi possível entrar em contato e administrar um levantamento longitudinal da reconstrução de trajetória e uma entrevista semiestruturada. Os resultados mais relevantes mostram que, quanto aos aspectos sociodemográficos, a maioria dos sujeitos chegou de outros locais para iniciar os estudos universitários, sendo que seus pais tiveram acesso a baixos níveis de escolaridade. Em relação à situação de emprego, o percentual de estudantes que relatou trabalhar e estudar em paralelo não é majoritário, porém, observa-se que, em algumas ocasiões, o momento do início das atividades de trabalho coincide com o do início dos estudos universitários. Finalmente, os eventos vitais que os alunos consideraram influentes em suas trajetórias educacionais são variados. No entanto, aprofundando os motivos que levaram esses jovens a abandonar seus estudos, constatamos que a maioria atribui o abandono a aspectos relacionados à escolha profissional e a dificuldades na ordem acadêmica.

Palavras chave: trajetórias acadêmicas; abandono; características sociodemográficas; família; trajetória de trabalho; acontecimentos vitais.

\section{Introducción}

No son pocos los jóvenes que año tras año ingresan al nivel superior. Sin embargo, apenas algo más de la mitad permanece más de un semestre o ciclo lectivo y, de los que quedan, muchos abandonan o se pierden en el camino (Coronado y Gómez Boulin, 2015). 
En carreras de ingeniería -que son las que interesan particularmente en este artículo-, con diferencias por región y por carrera, la deserción va del 30\% al 50\% en nuestro país y se concentra, principalmente, en los primeros años del cursado (Panaia, 2011; 2013). Se registran altas tasas de deserción precoz y temprana, esto es, entre el periodo de inscripción y el momento del ingreso, como así también durante los primeros meses de cursado (Sierra y Hernández, 2014).

En el sentido mencionado, estudios realizados con desertores de carreras de ingeniería en la Universidad Nacional de Río Cuarto (Argentina) -contexto en el que se enmarca el estudio aquí presentado- dieron cuenta de que un grupo mayoritario, aproximadamente un 40\%, permaneció en la carrera tan solo entre 1 y 6 meses y luego la abandonó; período demasiado corto como para generar experiencias y lograr aprendizajes. Otro grupo importante de sujetos (casi un 30\%) informó una permanencia de entre 7 meses y un año. Estos datos estarían mostrando que, para muchos sujetos, la decisión de abandonar los estudios se toma muy rápidamente, aun antes de haber tenido experiencias académicas de rendir parciales o finales. De hecho, la situación más frecuente entre los desertores es la de no haber aprobado ningún final en el marco de la carrera iniciada y luego abandonada (Chiecher, 2015).

Lejos de ser privativa de una universidad en particular, la preocupación por la calidad de la educación universitaria ofrecida y la disonancia entre un ingreso masivo y una escasa tasa de egreso afecta e inquieta a gran parte de las universidades argentinas y también de otros países. De hecho, un informe de la OIT (Organización Internacional del Trabajo) plantea que si bien el 65\% de la juventud de América Latina está cursando o ha llegado como nivel máximo de estudios a la educación secundaria, solamente el 14\% completa los estudios superiores (Coronado y Gómez Boulin, 2015). Las tasas pueden variar según países y regiones.

En el caso particular de las carreras de ingeniería encontramos que, en Argentina, se caracterizan, por lo general, por un decreciente número de inscriptos, un reducido número de graduados, una lentificación en el recorrido de los trayectos curriculares y un abandono marcado de los estudios (Aparicio, 2009; Chiecher et al., 2011; Falcone y Stramazzi, 2011; García et al., 2011; Panaia, 2011; Parrino, 2014).

Dadas las características multidimensionales y complejas de las trayectorias académicas es necesario estudiarlas con detenimiento y en profundidad para contribuir a la elaboración de orientaciones tendientes a promover una mayor calidad educativa así como la permanencia y el logro académico de los alumnos universitarios (Aparicio, 2009; Parrino, 2005).

Este estudio propone reconstruir retrospectivamente trayectorias estudiantiles de alumnos de ingeniería que abandonaron sus estudios, analizando qué factores influyeron en la decisión de abandonar, aspectos vinculados con el contexto familiar, la trayectoria laboral y la historia de vida de los sujetos.

\section{Encuadre teórico y antecedentes \\ Trayectorias académicas reales: demoras y abandono}

El sistema educativo se define a través de su organización, estructurada por distintos niveles, por la gradualidad del currículum y la anualización de los años de instrucción, todo lo que marca una trayectoria académica tipo. La trayectoria académica es entendida como aquel recorrido que realiza el estudiante dentro del sistema, el cual puede seguir la progresión lineal prevista -lo teóricamente esperable- o, por el contrario, desencauzarse, 
ocasionando una lentificación de los estudios o el abandono total del sistema (Terigi, 2009). En este trayecto que realiza el estudiante en el sistema educativo el aprendizaje va tomando sus propios tiempos ya que se ve atravesado por factores personales y del contexto, los que contribuyen a configurar un tipo particular de recorrido académico. Para referirse especialmente a este concepto Terigi (2010) diferencia las trayectorias académicas teóricas -aquellas que siguen los tiempos marcados por el sistema- de lo que son trayectorias académicas reales, es decir, aquellas que se distancian en mayor o menor medida de las teóricas e ideales puesto que se ven atravesadas por las historias de vida y las situaciones contextuales que a cada sujeto le toca vivir.

Ahora, en lo que refiere específicamente al recorrido que realizan los estudiantes en el nivel superior se constata que, en Argentina particularmente, no abundan las investigaciones sobre las trayectorias que siguen los alumnos que abandonan o retrasan sus estudios universitarios o que realizan cambios en su elección universitaria. En algunos trabajos se informan cifras, números y porcentajes de abandono pero resultan más escasas las investigaciones cuyo foco se centra en recuperar las voces, testimonios y experiencias de quienes ya no están en la universidad.

En el ámbito nacional emergen como interesantes antecedentes los estudios de Aparicio (2009) en relación a la demora en los estudios universitarios; los de Coronado y Gómez Boulin (2015) acerca de las trayectorias estudiantiles en sus distintas variantes, las trayectorias truncas y las de aquellos estudiantes que avanzan hacia la meta; los estudios realizados por Mastache, Monetti y Aiello (2014) también focalizados en las trayectorias de estudiantes universitarios, y finalmente, investigaciones sobre el abandono desarrolladas por Panaia (2013) y los grupos de investigación que dirige en distintas regiones del país. Aun así parece predominar cierto carácter punitivo sobre el abandono porque la meta a lograr es la carrera universitaria terminada y el resto de las trayectorias incompletas constituyen casos desviados del tipo ideal, representado por el graduado (Panaia, 2011). La propuesta de este trabajo es entonces contribuir al análisis de la problemática a la que se enfrenta la educación universitaria por las demoras en el cursado de la carrera y el marcado abandono. Para tal fin se propone la reconstrucción de trayectorias académicas de un grupo de estudiantes de ingeniería cinco años después de haber iniciado sus estudios universitarios, y que culminaron con el abandono.

Entendemos que el trabajo con trayectorias académicas permite construir un mapa complejo para su análisis por la multiplicidad de causantes que se entrelazan para generarlas y definirlas (Aparicio, 2009; Parrino, 2005).

\section{La significatividad del entorno: implicancias de las características socioeconómicas, laborales y vitales}

En la agenda de la psicología educacional cada vez cobran mayor fuerza las perspectivas socioculturales del aprendizaje, que lo conciben como un proceso social que no es independiente del contexto donde se produce.

Desde esta perspectiva, el aprendizaje tiene lugar en un contexto determinado y a través de actividades específicas que no son neutrales respecto de los resultados que se obtengan (Chiecher y Paoloni, 2009; Rinaudo, 2006; Rinaudo y Donolo, 2000). Es, además, meramente social y definido por las interacciones entre pares y con el ambiente donde se lleva a cabo, por lo cual la aproximación sociocultural al estudio de dichos procesos permite comprender qué relaciones se establecen con los escenarios culturales, la historia e incluso las mismas instituciones (Rinaudo, 2014). 
Apreciar la significatividad que tiene el entorno en los procesos de aprendizaje, es decir, todo aquello que sucede en paralelo al recorrido académico, permite entender al estudiante como un sujeto en desarrollo dentro de un contexto, que toma decisiones, lleva a cabo tareas, afronta diversos desafíos según la etapa de la carrera en que se encuentre, que hace de su trayectoria un recorrido que se va construyendo mientras se transita. Un recorrido que no es lineal sino que se va desplegando en una temporalidad que reúne pasado, presente y futuro (Greco y Nicastro, 2012, en Coronado y Gómez Boulin, 2015). Esta mirada amplia permite concebir a la trayectoria educativa en doble sentido: el teórico, ideal, establecido, subjetivo y sujeto a normas y legalidades que no siempre coinciden con el otro, el que finalmente vive y construye el sujeto (Greco y Nicastro, 2012, en Coronado y Gómez Boulin, 2015). Tener en cuenta este aspecto es fundamental para la interpretación de las historias de los sujetos, así como para comprender sus decisiones, sus metas, la manera en que se comportan y el modo en que enfrentan los desafíos.

¿Cuáles son los motivos por los que los estudiantes expresan haber decidido abandonar sus estudios? ¿De qué modo impacta en las trayectorias estudiantiles la necesidad o la decisión de trabajar en paralelo a los estudios? ¿Se observa relación entre el desempeño laboral y las trayectorias de abandono de los estudios? ¿Qué participación tienen ciertos acontecimientos vitales importantes en las trayectorias de los estudiantes? ¿De qué manera influyeron en la decisión de abandonar la carrera? Estos son algunos de los interrogantes que han orientado el presente estudio y a los que procuraremos dar respuesta en base a los datos recogidos.

\section{Metodología}

El estudio presentado se valió de métodos mixtos, apelando a la combinación de enfoques cuantitativos y cualitativos tanto para la recolección como para el análisis de los datos. Como se ha mencionado, cinco años después de su ingreso a la universidad se reconstruyen retrospectivamente trayectorias académicas de un grupo de estudiantes que abandonaron carreras de ingeniería. Fueron contactados con el objetivo de recoger datos y analizar la participación que en sus trayectorias y decisiones de abandono pudieron haber tenido aspectos vinculados con sus contextos e historias de vida.

Puntualmente, los objetivos que orientaron este trabajo fueron: 1) conocer las características sociodemográficas del estudiante y de su contexto familiar; 2) indagar acerca de la situación laboral paralela a los estudios; 3) conocer los acontecimientos vitales trascendentes en la vida del sujeto y su impacto en la decisión de desertar y, finalmente, 4) profundizar en el conocimiento de los motivos del abandono.

\section{El grupo objeto de estudio y la construcción de la población}

Participaron del estudio alumnos que en el año 2012 se habían inscrito para cursar alguna de las cuatro carreras de ingeniería que ofrece la Universidad Nacional de Río Cuarto, a saber: Ingeniería Mecánica, Ingeniería Electricista, Ingeniería Química e Ingeniería en Telecomunicaciones. En el momento de iniciar la investigación -año 2016- según lo estipulado en el plan de estudios, este grupo debía haber estado cursando el quinto año de la carrera elegida. Sin embargo se halló que 79 de 159 sujetos inscriptos (50\%) sí eran alumnos efectivos de la Facultad de Ingeniería en tanto que los 80 restantes (50\%) no habían renovado inscripción y no estaban activos en las carreras en que se habían inscripto cinco años antes, con lo cual fueron identificados como desertores. 
Este trabajo se enfoca en el grupo de 80 estudiantes que se constató habían abandonado la carrera. Se disponía de datos sociodemográficos del grupo completo $(\mathrm{N}=80)$. Tras un arduo trabajo de búsqueda y localización de los sujetos se logró también contactar y administrar los instrumentos de recolección de datos a 18 de ellos.

En este punto es importante destacar la complejidad del operativo de campo puesto que implicó la búsqueda de los sujetos y el intento de contacto con cada uno de ellos. En algunos casos los números telefónicos estaban desactualizados y entonces ni siquiera se consiguió localizarlos. En otros casos, tras varios intentos no se obtuvo respuesta alguna. Finalmente, algunos de los sujetos contactados manifestaron con total libertad no desear formar parte del estudio ya que la participación era voluntaria. Probablemente, los sinsabores y sentimientos de frustración que pudo haber dejado en muchos de estos chicos una experiencia universitaria truncada se relacionan con la negativa a ser entrevistados. Tanto la administración de la encuesta como la entrevista fueron llevadas a cabo vía telefónica puesto que, luego de haber abandonado sus estudios universitarios -en algunos casos hasta 4 años antes del comienzo del relevamiento- varios habían regresado a sus ciudades de origen o se encontraban realizando actividades alejadas de la universidad.

\section{Instrumentos de recolección de datos}

Se dispone, por un lado, de datos sociodemográficos de los 80 sujetos identificados como desertores, los que fueron extraídos del Sistema de Información de Alumnos de la Facultad de Ingeniería, Universidad Nacional de Río Cuarto.

Por otro lado, 18 de los 80 sujetos dieron respuesta a una encuesta longitudinal que permite una reconstrucción retrospectiva de sus trayectorias y a una entrevista semiestructurada orientada a recuperar los motivos del abandono desde las voces de sus protagonistas. La encuesta Iongitudinal, elaborada por Panaia (2006), se conforma de una serie de calendarios que permiten captar mes a mes y año a año las trayectorias académicas y laborales así como los principales acontecimientos vitales que afectaron al sujeto desde el ingreso a la universidad hasta el momento de abandonar los estudios. La entrevista, por su parte, se orientó a profundizar en los motivos del abandono.

La recolección de los datos fue llevada a cabo vía telefónica, las entrevistas realizadas fueron grabadas en audio, previo consentimiento del entrevistado a tal fin, y luego transcriptas en registros escritos para su análisis. En los siguientes apartados se presentarán los principales resultados hallados luego de la administración de los instrumentos de recolección de datos antes consignados.

\section{Resultados}

\section{Características sociodemográficas del grupo total de alumnos desertores de la cohorte 2012}

\section{Edad}

La edad promedio de los estudiantes al inicio del relevamiento era de 24 años. La media es un poco mayor respecto del grupo de alumnos de esta misma cohorte que continuaba sus estudios, cuyo promedio era de 22 años (Moreno y Chiecher, 2018). Quizá se trate de estudiantes que por alguna razón demoraron en comenzar los estudios luego de haber terminado el nivel medio o estudiaron alguna otra carrera antes de inscribirse en la Facultad de Ingeniería. 


\section{Sexo}

Si observamos la distribución por sexo en el grupo de desertores $(\mathrm{N}=80)$, vemos que son mayoritariamente varones (91\%). Cabe señalar que también son mayoritariamente varones los ingresantes en carreras de ingeniería (82\%). No obstante, la representatividad de los varones es mayor en el grupo de quienes abandonaron, lo que sugiere que los varones podrían propender más que las mujeres a abandonar los estudios.

El Gráfico 1 presenta comparativamente la distribución según sexo en el grupo total de ingresantes (159) y en el grupo de desertores (80).

Gráfico 1. Distribución de los estudiantes por sexo, en alumnos desertores y grupo total de ingresantes 2012

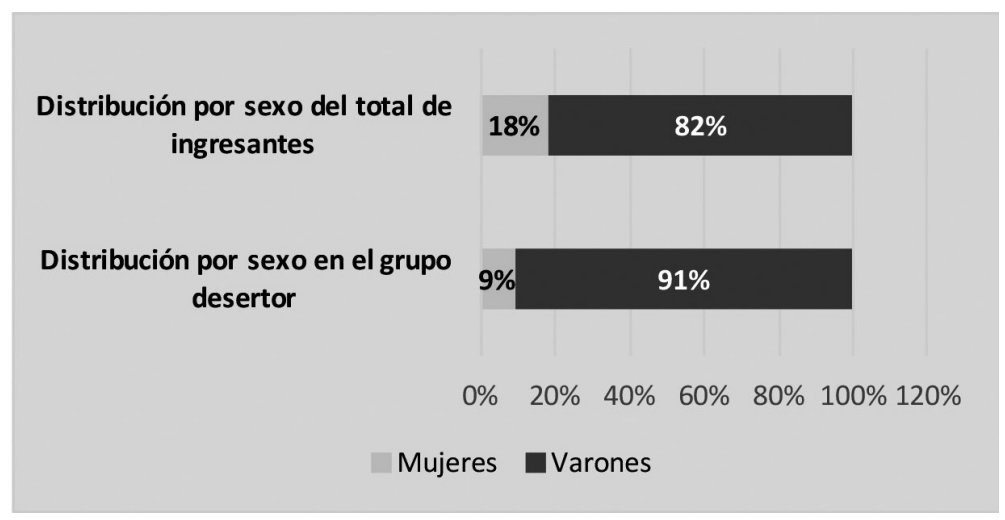

Fuente: elaboración propia

\section{Procedencia}

En cuanto a esta variable relativa a la procedencia de los estudiantes, en el Gráfico 2 se puede observar que el mayor porcentaje de alumnos desertores se ha trasladado de otras localidades para iniciar sus estudios universitarios. De hecho, la Universidad Nacional de Río Cuarto recibe cada año a muchos estudiantes que vienen de la zona, o incluso de provincias más alejadas para cursar sus estudios.

Gráfico 2. Distribución de los estudiantes desertores según la procedencia $(N=80)$

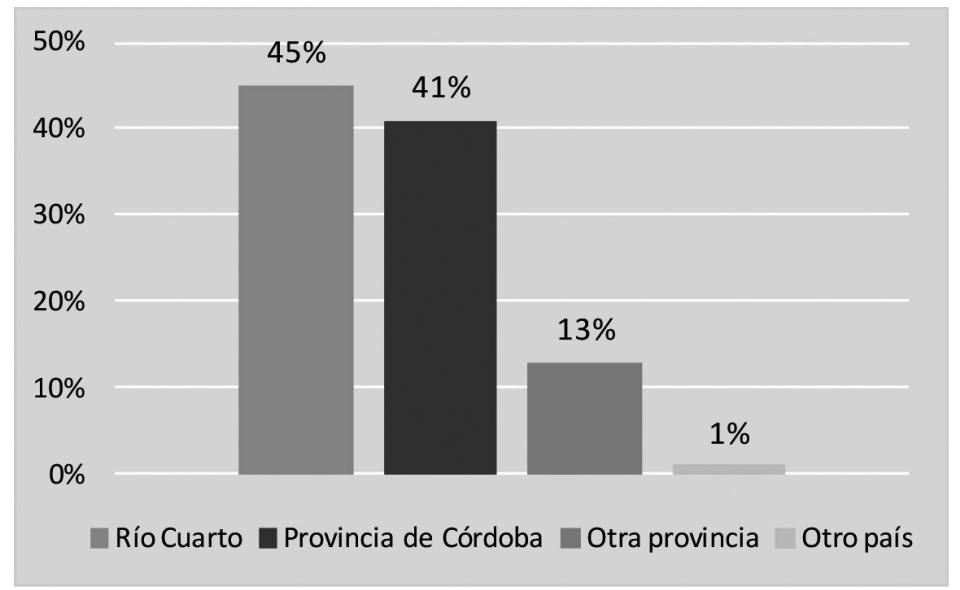

Fuente: elaboración propia 
Tal como se observa, más de la mitad de los estudiantes que abandonaron sus estudios (55\%) provenía de otros puntos del país e incluso de otros países. Esta es una variable importante si se tiene en cuenta que son estudiantes que en su mayoría han debido cambiar de ciudad, alejarse del seno familiar, aprender y acostumbrarse a usar medios de transporte distintos, compartir la vivienda con desconocidos o vivir solos, realizar tareas domésticas, etc. Estos obstáculos que atraviesan quienes comienzan los estudios en una ciudad diferente a la de origen los coloca en una situación de mayor vulnerabilidad respecto a quienes pueden estudiar permaneciendo en la ciudad en la que viven y cerca del hogar familiar. De hecho, en estudios previos con alumnos de esta misma cohorte pero cuyas trayectorias académicas se ajustaban al recorrido teórico o ideal, se encontró que un $60 \%$ eran oriundos de Río Cuarto (Moreno y Chiecher, 2018).

\section{Nivel educativo los padres}

Al analizar el nivel educativo del padre y de la madre de los sujetos que abandonaron sus estudios se observan datos que respaldan resultados de investigaciones previas. En general se trata de estudiantes universitarios de primera generación, es decir, cuyos padres no accedieron mayormente a estudios superiores por lo que estos jóvenes son los primeros en la familia en llegar a la universidad: el $80 \%$ de los padres y el $70 \%$ de las madres no habían transitado el nivel de educación superior. El Gráfico 3 muestra de manera desglosada el nivel educativo alcanzado por los padres.

Gráfico 3. Nivel educativo del padre y de la madre $(\mathrm{N}=80)$

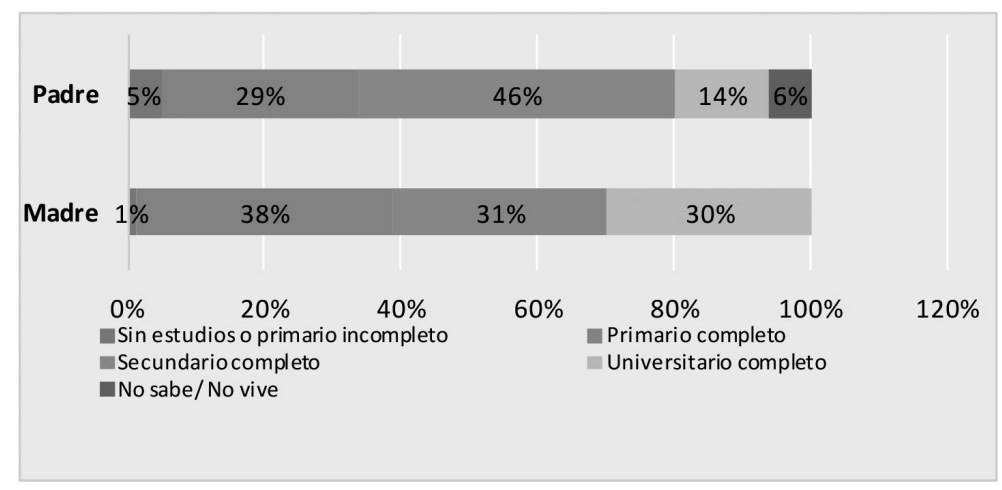

Fuente: elaboración propia

Se puede observar que el $80 \%$ de los padres no ha tenido acceso a estudios de nivel terciario o universitario. El $46 \%$ ha completado el nivel secundario y el $29 \%$ ha completado solo la educación primaria. Las madres tienen un mayor porcentaje en el nivel de instrucción terciaria y universitaria comparado con los padres. Sin embargo, solo un $38 \%$ completó la primaria y un $31 \%$ el nivel secundario.

Estos resultados parecen estar en consonancia con los hallados en otros estudios, donde la familia y el ambiente en que transcurre la vida de los sujetos parecen tener un peso particular sobre el rendimiento (Aparicio, 2009). Particularmente el rol de las madres se señala como fundamental. En diversos estudios se ha demostrado la incidencia positiva que tienen los altos niveles de instrucción en las madres en el rendimiento de los hijos ya que parecen tener actitudes positivas hacia el estudio de sus hijos, se preocupan por su desempeño e incluso los orientan en la continuación de los estudios hasta su titulación (Garbanzo, 2007). Este estudio ratifica de alguna manera esos resultados. 


\section{Actividad laboral de los padres}

Se constató que el mayor porcentaje de los padres de los desertores trabajan (ver Gráfico 4).

Gráfico 4. Actividad laboral del padre y de la madre $(\mathrm{N}=80)$

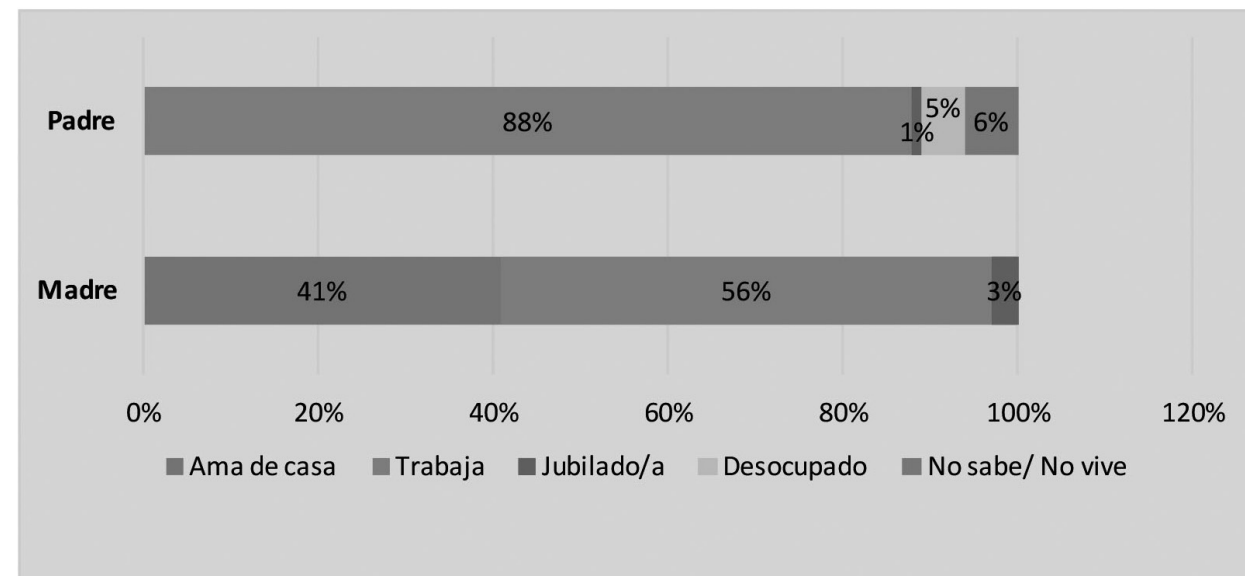

Fuente: elaboración propia

Las madres mayormente trabajan y lo hacen fuera del hogar (56\%). Entre los ámbitos laborales más destacados se encuentran los servicios públicos o privados, docencia y comercio, mientras que un $41 \%$ se dedica a las tareas del hogar (amas de casa).

En el caso de los padres encontramos que también la mayoría trabaja (88\%). Entre los ámbitos laborales más destacados se encuentran servicios públicos o privados, industria y construcción, agricultura y ganadería. Se observa también un 5\% de los padres en condición de desocupados.

Comparado con el grupo de estudiantes de la misma cohorte que han logrado trayectorias académicas exitosas, el porcentaje de madres que son amas de casa se duplica entre los desertores, no así el de los padres (Moreno y Chiecher, 2018). Posiblemente esta diferencia observada en los porcentajes de ocupación respecto del padre y de la madre en este grupo desertor (88\% y 56\% respectivamente) esté indicando que entre los padres de los desertores es más habitual que solo uno de los progenitores opere como sostén del hogar mientras que la situación se presenta diferente entre los estudiantes que transitaron las trayectorias teóricas tal como las estipula el plan de estudios. En estos casos, mayormente los dos padres trabajan y obtienen remuneración por ello, lo que podría representar mejores condiciones económicas en estas familias.

\section{Trayectorias laborales paralelas a los estudios}

En cuanto a las trayectorias laborales que los desertores transitaron en paralelo a los estudios se recuperaron datos de las 18 personas contactadas. El Gráfico 5 muestra que un 39\% compatibilizó estudio y trabajo en algún momento de su paso por la universidad. Considerando que entre los estudiantes de la misma cohorte que habían logrado cursar en los términos teóricos estipulados el porcentaje de los que alguna vez trabajaron fue de 53\% (Moreno y Chiecher, 2018), el resultado no conduciría a suponer que las actividades laborales hayan estado entre las principales razones por las cuales estos jóvenes decidieron abandonar los estudios. Sin embargo, lo que sí podemos señalar es que -a diferencia de los resultados hallados con el grupo de sujetos que seguían en la universidad- el momento 
en el que las actividades laborales aparecen para este grupo de desertores es en el inicio del cursado. Es decir, que son estudiantes que comienzan los estudios universitarios con actividades laborales paralelas, lo que implica ciertos obstáculos adicionales que se suman al esfuerzo y al reajuste necesarios para adaptarse al ritmo de la universidad. Se complejizan así situaciones tales como el tiempo de dedicación al estudio y las posibilidades de cursar frente a bandas horarias extensas. No es el caso de aquellos estudiantes que logran llevar sus estudios a término o presentan demoras menores y cuyas actividades laborales, si es que trabajan, aparecen en los últimos años del cursado y suelen, además, estar vinculadas con sus estudios (Moreno y Chiecher, 2018).

Gráfico 5. Compatibilización entre estudio y trabajo $(\mathrm{N}=18)$

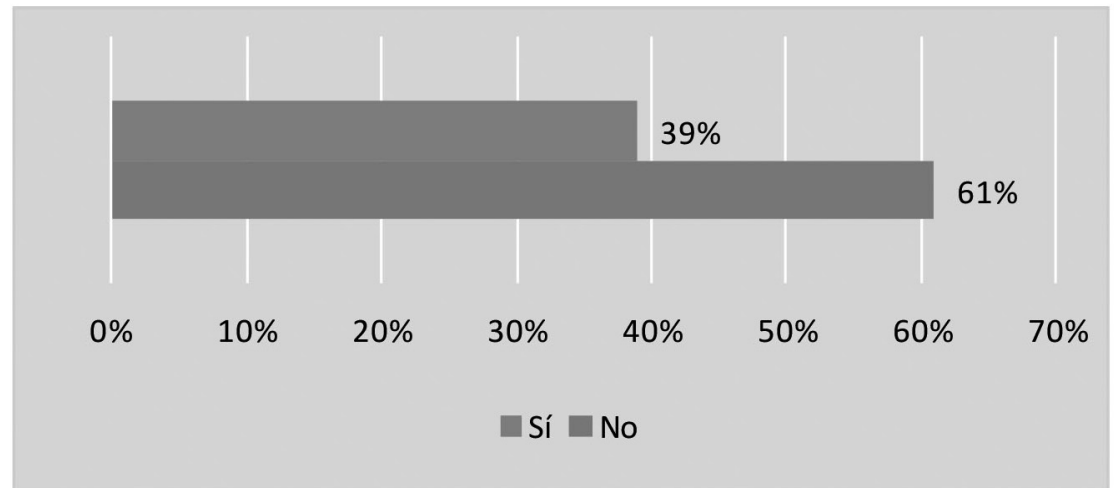

Fuente: elaboración propia

Otro aspecto a destacar son las características o contenidos de los trabajos que informaron haber realizado los sujetos entrevistados mientras estudiaban. La mayoría se desempeñó en trabajos informales, inestables y temporales, en comercios o por cuenta propia (changas), con escasa vinculación con la carrera que estudiaban. Esta es otra consideración importante ya que las actividades laborales son necesarias y casi un medio para sostener sus estudios. Cabe destacar que de los sujetos entrevistados solo el $22 \%$ accedió a una beca de ayuda económica la que, a su vez, se conserva con el buen rendimiento, aspecto que no favorece precisamente a grupos con estas características cuyos porcentajes de aprobación durante el primer y segundo año suelen ser muy bajos.

\section{Acontecimientos vitales trascendentes}

Finalmente, otro de los datos recabados fue el vinculado a los acontecimientos vitales trascendentes, especialmente aquellos que los sujetos reconocen como influyentes en sus trayectorias académicas, tal como se evidencia en el Gráfico 6. 
Gráfico 6. Acontecimientos vitales trascendentes $(\mathrm{N}=18)$

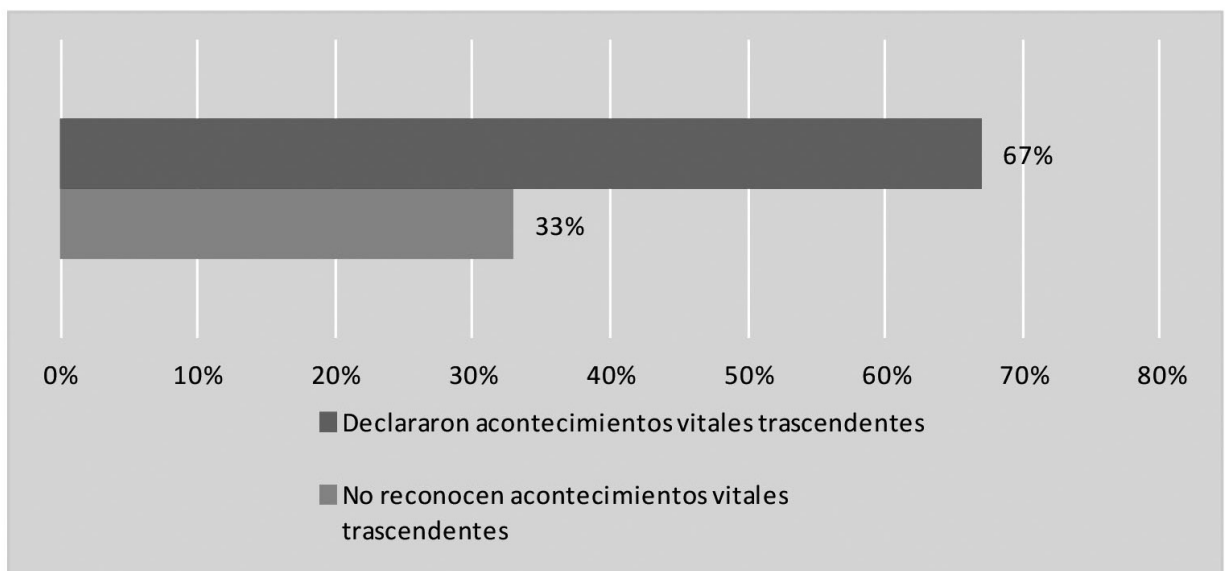

Fuente: elaboración propia

Como es esperable para este grupo, la mayoría de los entrevistados (67\%) expresó haber atravesado algún suceso de índole personal, familiar o económica e incluso problemas de salud que de alguna manera impactaron en el recorrido de su trayectoria académica. Veremos en los próximos apartados qué vinculaciones existen entre estos acontecimientos y el desenlace del abandono.

\section{Acerca de los motivos del abandono. Las voces de los estudiantes}

En el Gráfico 7 pueden observarse los años en los que se registran los mayores porcentajes de abandono en el grupo total de desertores de la cohorte analizada $(\mathrm{N}=80)$, desde el momento de ingreso a la universidad hasta el año de comienzo del relevamiento (2016). De acuerdo con lo estipulado en el plan de estudios es en este año cuando se supone que estos estudiantes deberían estar cursando el quinto año de la carrera.

Gráfico 7. Porcentajes de abandono por año de la cohorte 2012, desde el momento del ingreso hasta $2016(\mathrm{~N}=80)$

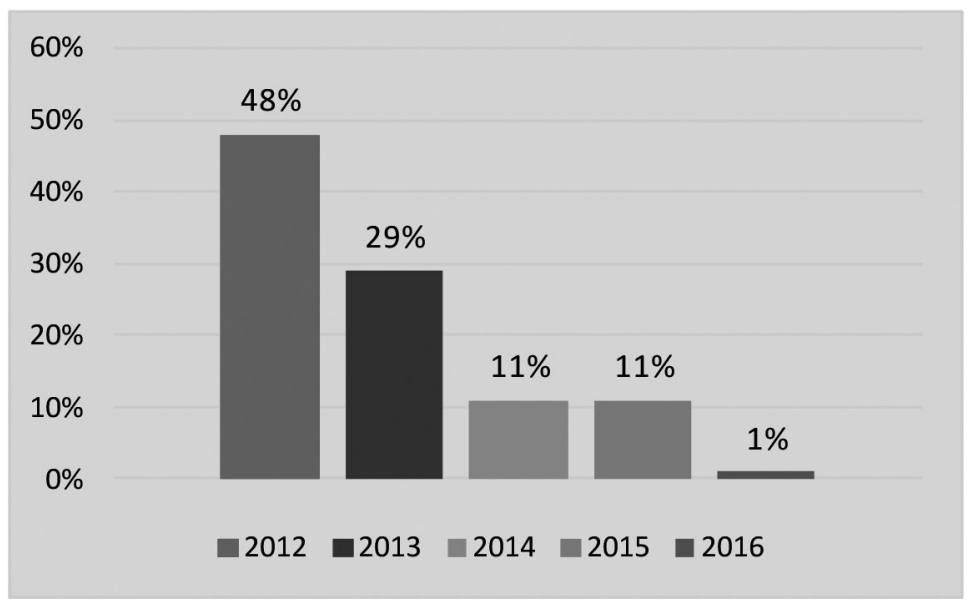

Fuente: elaboración propia 
Como es esperable, en los años 2012 y 2013 -que corresponden al primer y segundo año en la universidad- se observan los mayores porcentajes de abandono (48\% y $29 \%$ respectivamente). Es el momento que corresponde al inicio de la vida universitaria. En el caso de los 18 sujetos entrevistados, poco más del 50\% abandonó sin haber aprobado ninguna materia de las que corresponden al primer año de cursado. Esto es sumamente interesante si pensamos el ingreso a la universidad como un momento clave, donde probablemente se definen muchas de las trayectorias académicas -que en el caso del grupo que estudiamos culminaron con el abandono- y donde seguramente muchas de las razones se relacionen con que los estudiantes se hayan encontrado más vulnerables e incluso desprovistos de los recursos necesarios para afrontar esa nueva etapa.

Al indagar mediante las entrevistas las razones por las cuales decidieron abandonar la carrera, los sujetos expresaron los siguientes motivos -ver Gráfico 8- (categorías mutuamente excluyentes):

Gráfico 8. Motivos del abandono $(\mathrm{N}=18)$

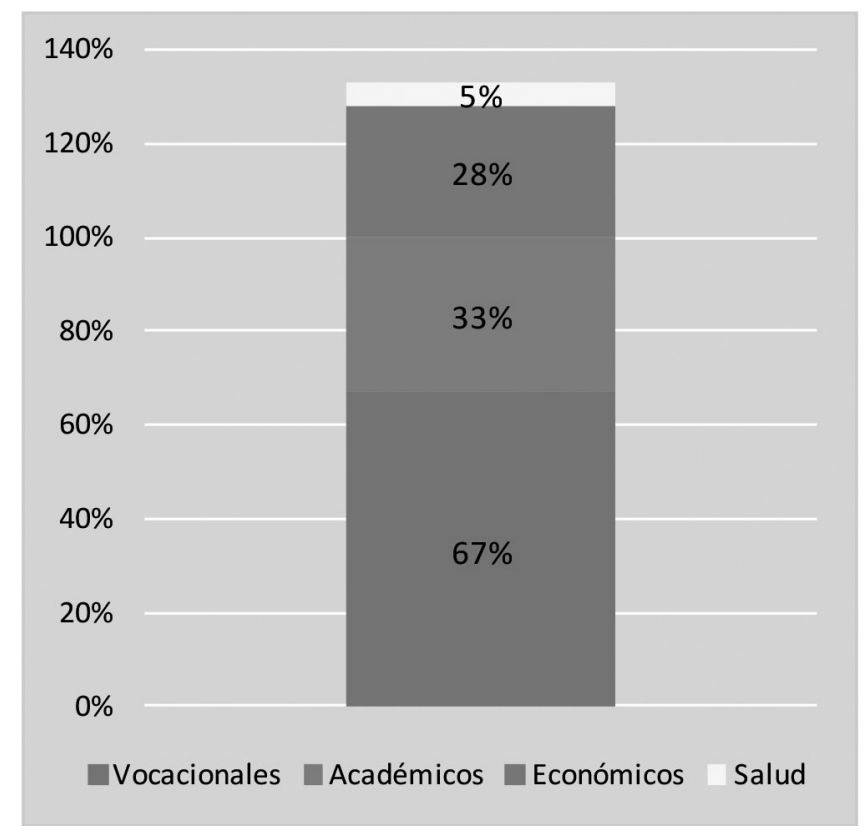

Fuente: elaboración propia

A continuación se describen las categorías enunciadas, las que aluden a los motivos de abandono que esgrimieron los sujetos entrevistados.

Motivos vocacionales. El $67 \%$ de los sujetos afirmó que la carrera no respondió a las expectativas iniciales, que no era lo esperado. Muchos se inscribieron sin conocer demasiado qué iban a estudiar puntualmente o cuáles eran los ámbitos laborales de desempeño de la profesión e incluso con representaciones erróneas de la disciplina elegida. Otros se inscribieron en alguna de las ingenierías porque era la carrera "más parecida" a la que hubiesen querido estudiar. El 50\% de los entrevistados que expresaron haber abandonado la ingeniería por motivos vocacionales, en el momento de ser entrevistados se encontraban cursando otras carreras y hasta en algunos casos finalizándolas. Mientras tanto, otros ya se habían graduado en otras especialidades. La otra mitad optó por trabajar, en algunos casos tras haber intentado cursar otra carrera. Veamos algunos ejemplos en palabras de los entrevistados: 
“La verdad es que estaba muy convencido de qué quería estudiar y qué no quería estudiar, así que no me fue muy complicado, fue directa la decisión... Me fui dando cuenta, cuando empecé, de que no me gustaba, tampoco estuve tanto tiempo, fueron 3 meses los que habré cursado ingeniería... dejé y me inscribí en el 2014 en el profesorado [de Química]" (Desertor de Ingeniería Química)

"Sí, cuando pasó el año, o sea cuando comencé a estudiar $2 .^{\circ}$ empecé a dudar porque ya empecé a pensar en el futuro yo trabajando de ingeniero electricista, dedicándome a trabajar en ingeniería, en una empresa, en el lugar que me tocara, y veía que no me llamaba mucho la atención. Además de eso no me sentía cómodo o contento imaginando trabajar de eso..." (Desertor de Electricista).

"Ya hacía un tiempo que me venía planteando que no era lo mío, no me sentía cómodo con las materias que estudiaba, entonces hablé con mi familia y me dijeron que si era eso, que probara con otra carrera pero que siguiera... me dijeron 'cambiate, pero cambiate ya, para no perder más tiempo, si no ya está"' (Desertor de Mecánica).

"En el primer cuatrimestre me fue, para mí, súper bien, pero después sentí, como que me gustaría estudiar otra cosa..." (Desertor de Telecomunicaciones).

Motivos académicos. El 33\% de los sujetos ofreció motivos académicos como explicación de la deserción y probablemente como el detonante del resto de los motivos que aquí se mencionan. El no poder adaptarse a las altas exigencias académicas, las extensas horas de cursado, la insuficiente base que traen desde el secundario, el no poder organizar las estrategias de estudio -y como consecuencia desaprobar exámenes- pareciera haberlos desbordado, viéndose entonces desprovistos de las herramientas necesarias para afrontar esta nueva etapa. A continuación se transcriben algunos fragmentos de entrevista que ilustran esta categoría de respuestas:

"Sabía que las ingenierías eran muy difíciles pero me gustaban mucho los números y quise probar, pero el primer año se me hizo muy difícil y no seguí, por ahí tendría que haber seguido..." (Desertor de Mecánica).

"Estoy laburando, tengo un negocio de electricidad acá en el pueblo que abrimos con mi viejo... fue medio así, se decidió, ya que no me iba muy bien, partí para otro lado... Ya para retomar la ingeniería estoy bastante lejos pero no por motivos de la universidad, es que no me senté nunca a estudiar, me costaba, muy vago... por eso nada más..." (Desertor de Electricista)

Motivos económicos/laborales. Un 28\% de los sujetos manifestó no tener muchas opciones, sobre todo quienes provenían de otras localidades. Si no aprobaban o no encontraban una carrera alternativa -que en muchos casos comenzaron y también abandonaron- tenían que salir a trabajar. En muchos de los casos el hecho de tener que trabajar implicó alejarse definitivamente de los ámbitos académicos.

“La principal razón es la económica. Si bien en mi trabajo yo tenía cierta flexibilidad, por ahí lo que se complicaba o lo que yo consideraba como un punto en contra en la carrera es que, por ejemplo, teníamos siempre clases a la tarde y a lo mejor los horarios de consulta eran siempre a la mañana o alguna prueba a la mañana, entonces, si vos tenías siempre a la tarde programabas el trabajo a la mañana y a lo mejor un día te decían, che chicos tienen que venir a rendir, el lunes a las 9, a tal pabellón. Eso lo veía como un punto muy negativo de la universidad o sea, para el chico que trabaja no está pensado..." (Desertor de Electricista).

"Me salió un trabajo, era buen trabajo y qué se yo, decidí dejar para irme a trabajar... ya no tengo tiempo... o sea, lo que es ingeniería, tiene mucha carga horaria y aparte precisa mucho tiempo la carrera en casa, para estudiarla..." (Desertor de Telecomunicaciones). 
Motivos de salud. Finalmente, un 5\% de los sujetos argumentó tener que afrontar problemas de salud mental (depresión), por lo que se vio limitado en muchas actividades de su vida cotidiana, entre ellas los estudios.

“Me gusta la carrera de ingeniería en general... la dejé en ese momento por unas cuestiones personales mías... Fueron cosas que empezaron a pasarme a nivel personal que me llevaron a una depresión y a dejarla y también tuve algunos problemas de salud y bueno, me vi forzado a dejarla..." (Desertor de Electricista).

\section{Apreciaciones finales}

Este artículo procura contribuir a la comprensión de aquellos factores asociados al abandono de la carrera universitaria. Así, el estudio propone la reconstrucción retrospectiva de las trayectorias académicas de un grupo de estudiantes de ingeniería pertenecientes a la cohorte 2012, quienes fueron contactados para la recolección de datos cinco años después de su ingreso y que, en el momento de inicio del relevamiento se constató que habían abandonado sus estudios.

Se analiza, por un lado, el impacto de los aspectos sociodemográficos tales como la edad, el sexo, la procedencia, los niveles educativos y ocupación de los padres, así como también las actividades laborales desarrolladas por los sujetos en paralelo al tiempo de cursado de la carrera y a los acontecimientos trascendentes en sus vidas que pudieron haber tenido algún impacto en el desempeño académico. Por otro lado se profundiza en las razones del abandono desde la perspectiva de los estudiantes que fueron entrevistados.

Entre los aportes más relevantes podemos destacar que quienes abandonaron eran, en su mayoría, estudiantes que llegaron de otras localidades y se instalaron en la Ciudad de Río Cuarto para iniciar sus estudios universitarios.

En cuanto a sus padres, la mayor parte no ha tenido experiencias en el nivel de educación superior, por lo que estos jóvenes eran la primera generación de estudiantes universitarios en sus familias. Altos porcentajes indican que ambos progenitores trabajaban en el momento en que estos estudiantes estaban en la universidad y fundamentalmente era el padre el sostén del hogar.

El 39\% de los sujetos que abandonaron sus estudios indicó haber compatibilizado estudio y trabajo durante el cursado de la carrera. Si nos detenemos en este grupo encontramos, comparado con otros estudios, un punto interesante en el momento en que inician las actividades laborales. Estos estudiantes comienzan sus estudios cuando ya se encontraban insertos en algún trabajo, situación que los colocaría en cierta desventaja puesto que los obstáculos y limitaciones que deben enfrentar son mucho más numerosos que los que afrontan quienes no se ven en la necesidad de trabajar para solventar sus estudios: dificultades por las extensas horas de cursado y exigencias académicas extra áulicas, todo lo que se suma a lo que implica adaptarse a una nueva institución, con normas propias, un lenguaje particular y espacios desconocidos.

El 67\% de los entrevistados informó haber atravesado durante el período que cursaba la carrera algún acontecimiento vital que impactó en su desempeño académico, ya sea de índole personal, familiar, económica e inclusive de salud. Sin embargo, profundizando en los motivos que llevaron a estos jóvenes a abandonar sus estudios encontramos que la mayoría lo atribuyó a aspectos más personales vinculados con una errónea elección vocacional, con el desencanto por la carrera elegida, las dificultades en el estudio y la insuficiente base de conocimientos que traen desde el nivel medio, expresado dentro de los motivos académicos. 
Los resultados hallados -que muestran además altos porcentajes de abandono en los primeros años de carrera- representan un llamado de atención a colocar la mirada en el momento del ingreso universitario, e incluso en el trabajo en conjunto con instituciones de nivel medio. No es arbitrario que los mayores porcentajes de abandono se presenten en el primer y segundo año de la carrera, representado en más del 75\%.

Aunque es frecuente que se responsabilice al estudiante por los buenos o malos resultados obtenidos, no podemos separar los aprendizajes del ámbito contextual situacional donde se llevan a cabo. Las trayectorias académicas que los estudiantes transitan no son solo resultado del esfuerzo que realizan o del compromiso individual que asumen sino que también se ven influidas por las historias de vida y otras trayectorias -como las laborales, por ejemplo- que se cruzan con las académicas. Si se observan los aprendizajes desde esta perspectiva contextual las instituciones educativas tienen mucho por ofrecer para influir de manera positiva en las experiencias de aprendizaje de los estudiantes y promover así trayectorias académicas de logro que garanticen la permanencia en la universidad.

Claramente, una de las mayores dificultades que presentan estos alumnos ha sido la imposibilidad de poder regular sus propios procesos de aprendizaje. Muchos estudiantes se reconocieron a sí mismos en ese momento como inexpertos. Eran incapaces de organizar sus actividades académicas, se proponían metas u objetivos y los posponían, no estudiaban lo suficiente, no dedicaban el tiempo necesario, se distraían con mucha facilidad, leían poco y no comprendían, utilizaban técnicas de estudio repetitivas, les costaba armar buenos grupos de estudio, entre otras, sin contar los aspectos afectivos y emocionales que atravesaban por esa nueva etapa de vida, el desconocimiento del manejo institucional -como, por ejemplo, no saber que una materia podía ser correlativa de otra al año siguiente- o la situación laboral.

Los aprendizajes en la universidad requieren del manejo de determinadas habilidades, estrategias, destrezas y conocimientos que son específicos de las disciplinas elegidas y necesariamente se aprenden en la misma experiencia. Sin embargo, lo que sí se podría potenciar desde la universidad y especialmente enseñar a los alumnos que ingresan, es a desarrollar un control interno en los modos de aprender, a tener cierta evaluación crítica del conocimiento transmitido y a reforzar habilidades de meta estudio. Y lo óptimo sería comenzar a trabajar con estos recursos en instancias anteriores del sistema educativo (Blakemore y Frith, 2000 en De la Barrera y Donolo, 2009).

Si bien cada ingresante llega a la universidad con determinadas concepciones acerca de lo que implica aprender, es posible diseñar propuestas desde el contexto de la universidad que favorezcan el logro de trayectorias académicas más exitosas. Estudios como el que presentamos, con una mirada de los factores que rodean al estudiante, pueden convertirse en los marcos de referencia para que desde las instituciones se tomen decisiones y se formulen programas que permitan abordar problemáticas como el ingreso, permanencia y egreso de los estudiantes universitarios. 


\section{Referencias bibliográficas}

Aparicio, M. (2009). La demora en los estudios universitarios. Causas desde una perspectiva cuantitativa. Mendoza: EDIUNC.

Casullo, M.M. et al. (1996). Proyecto de vida y decisión vocacional. Buenos Aires, Barcelona, México: Editorial Paidós.

Chiecher A. y Paoloni, P. (2009). Graduados de Ingenierías de la UNRC. Características estructurales, trayectorias educativas e itinerarios laborales. Universidad Nacional de Río Cuarto. Facultad de Ingeniería.

Chiecher A., Paoloni, P. y Guebara, J. (2011). Abandonadores de las carreras de ingeniería. Motivo de abandono de los estudios y definición de nuevas metas. Documento de Trabajo, N. ${ }^{\circ}$ 10. Río Cuarto, Universidad Nacional de Río Cuarto, Facultad de Ingeniería. Disponible en: http://www.ing.unrc.edu.ar/laboratorios/mig_rio4/archivos/10_documento-final.pdf

Chiecher A. (2015). Ingreso universitario y prevención del abandono. Usos posibles y potencialidades de los contextos virtuales. En Panaia, M. (Coord.) Universidades en cambio ¿Generalistas o profesionalizantes? Buenos Aires: Editorial Miño y Dávila.

Coronado, M. y Gómez Boulin M.J. (2015). Orientación, tutorías y acompañamiento en Educación Superior. Análisis de trayectorias estudiantiles, los jóvenes ante sus encrucijadas. Colección Universidad. Editorial Noveduc.

De La Barrera, M.L. y Donolo, D. (2009) Neurociencias y su importancia en contextos de aprendizaje. Revista Digital Universitaria. № 4. Vol. 10. Recuperado de: http://www.revista. unam.mx/vol.10/num4/art20/art20.pdf

Falcone, L. y Stramazzi, M. (2011). La medición de la deserción real y la actividad universitaria. En Martínez, S. (Comp.) Democratización de la universidad. Investigaciones y experiencias sobre el acceso y la permanencia de los/las estudiantes. Neuquén: Universidad Nacional de Comahue.

Ferrari, L. (1995). Cómo elegir una carrera. Opciones para pensar y decidir tu futuro. Buenos Aires: Editorial Planeta.

Garbanzo Vargas, G. (2007). Factores asociados al rendimiento académico en estudiantes universitarios, una reflexión desde la calidad de la educación superior pública. Revista Educación. Vol. 31, núm. 1, 2007, pp. 43-63. Universidad de Costa Rica. Disponible en: http:// www.redalyc.org/articulo.oa?id=44031103

García, J.C., González, M. y Zanfrillo, A. (2011). Desgranamiento universitario: perspectiva estudiantil en ingeniería. XI Coloquio Internacional de Gestión Universitaria de América del Sur (8 al 10 de diciembre de 2011). Universidad de Santa Catarina, Brasil.

Mastache, A., Monetti, E. y Aiello, B. (2014). Trayectorias de estudiantes universitarios: recursos para la enseñanza y la tutoría. Buenos Aires: Noveduc. 
Moreno, J. y Chiecher A. (2018). Demoras y trayectorias desacopladas en Ingeniería. Condicionantes sociodemográficos, laborales y vitales. IV Congreso Argentino de Ingeniería, $X$ Congreso Argentino de Enseñanza de la Ingeniería, 19 al 21 de septiembre de 2018. Córdoba, Argentina.

Panaia, M. (2006). Trayectorias de ingenieros tecnológicos. Graduados y alumnos en el mercado de trabajo. Buenos Aires: Editorial Miño y Dávila.

Panaia, M. (2011). Dejar la universidad ¿Decisión o imprevisto? Boletín Itinerarios, N 12. Disponible en: http://www.ing.unrc.edu.ar/laboratorios/mig_rio4/archivos/12boletinjunio-2011.pdf

Panaia, M. (2013). Abandonar la universidad ¿Decisión premeditada o imprevista? En Panaia, M. (Coord.) Abandonar la Universidad con o sin título. Buenos Aires: Editorial Miño y Dávila.

Parrino, M.C. (2005). Aristas de la problemática de la deserción universitaria. V Coloquio Internacional sobre Gestión en las Universidades de América del Sur. 3 al 5 de diciembre de 2005. Universidad Nacional de Mar del Plata.

Parrino, M.C. (2014). Factores intervinientes en la deserción universitaria. Revista Argentina de Educación Superior, año 6, N. ${ }^{\circ}$ 8, pp 39-61. Recuperado de: file:///C:/Users/Anal\%C3\%ADa/ Downloads/DialnetFactoresIntervinientesEnEIFenomenoDeLaDesercionUni4753784.pdf

Rinaudo M.C. y Donolo, D. (2000). Casandra y la educación. La universidad como contexto de aprendizaje. En Guerci de Siufi, B. (Comp.) Pensando la Universidad. Ed. Universidad Nacional de Jujuy.

Rinaudo, M.C. (2006). Estudios sobre lectura. Aciertos e infortunios en la investigación de las últimas décadas. Asociación Internacional de Lectura, Lectura y Vida. Buenos Aires.

Rinaudo, M.C. (2014). Estudios sobre el contexto de aprendizaje: arenas y fronteras. En P.V. Paoloni, M. Rinaudo y A. González Fernández. Cuestiones en psicología educacional: aspectos teóricos, metodológicos y estudios de campo. Tenerife: Sociedad Latinoamericana de: Comunicación Social (SLCS). Recuperado de: https://issuu.com/revistalatinadecomunicacion/ docs/cde01

Sierra, H. y Hernández, O. (2014). Sistema de alertas tempranas como herramienta de innovación tecnológica en la Universidad Santo Tomás para el fortalecimiento de la permanencia estudiantil y graduación oportuna. IV Conferencia Latinoamericana sobre Abandono en Educación Superior. Medellín.

Terigi, F. (2009). Las trayectorias escolares. Del problema individual al desafío de política educativa. Proyecto hemisférico "Elaboración de políticas y estrategias para la prevención del fracaso escolar". Ministerio de Educación de la Nación. Disponible en: http://www.bnm. me.gov.ar/giga1/documentos/EL004307.pdf

Terigi, F. (2010). Las cronologías de aprendizaje: un concepto para pensar las trayectorias escolares. Conferencia online. Recuperado de: http://www.chubut.edu.ar/concurso/ material/concursos/Terigi_Conferencia.pdf 


\section{Notas}

*Universidad Nacional de Río Cuarto, Córdoba (Argentina).

${ }^{1}$ Este estudio ha sido realizado con el apoyo financiero de una beca doctoral otorgada por el Consejo Nacional de Investigaciones Científicas y Técnicas (CONICET) de la República Argentina.

${ }^{2}$ Licenciada en Psicopedagogía, Universidad Nacional de Río Cuarto (Argentina). Beca doctoral, Consejo Nacional de Investigaciones Científicas y Técnicas (CONICET) (Argentina). Adscripta, Universidad Nacional de Río Cuarto.

${ }^{3}$ Doctora en Psicología, Universidad Nacional de San Luis (Argentina). Magíster en Educación y Licenciada en Psicopedagogía, Universidad Nacional de Río Cuarto (Argentina). Investigadora Independiente, Consejo Nacional de Investigaciones Científicas y Técnicas (CONICET).

\section{Contribución de autoría:}

Las autoras han realizado el artículo en partes iguales. 